\title{
Sources of Psychological Support for East Asians Reporting a Low Level of Mental Health
}

\author{
Stephen P. Wong and Cass Dykeman ${ }^{1}$ \\ Oregon State University \\ 104 Furman Hall \\ Corvallis, OR 97331-3502.
}

\section{A Preprint}

\begin{abstract}
Most research about preferred sources of mental health assistance has been completed in the West. Mental health research with non-Western cultures is needed. Using data from the East Asian Social Survey (EASS), this study examined help-seeking preferences for individuals with low mental health in four countries - China, Japan, South Korea, and Taiwan. Preferences for kin versus non-kin support, use of alternative medicine, and professional mental health assistance were examined, as were betweencountry differences in support preferences. The results align with previous research in East Asian countries that found a strong preference for using kin support to address mental health concerns, followed by non-kin support (i.e., close friends and co-participants in religious institutions), alternative medicine, and professional mental health services, respectively. While there is a strong preference for family support, many East Asians are open to seeking formal or informal mental health support outside the family. There were some significant differences between countries, with South Koreans being more likely to seek non-kin support and professional support for mental health concerns than Chinese. These differences are discussed in the context of cultural and policy developments in East Asian countries.
\end{abstract}

Keywords: East Asian, family support, mental health, alternative medicine

\footnotetext{
${ }^{1}$ Correspondence concerning this article should be addressed to Cass Dykeman at dykemanc@oregonstate.edu
} 


\section{Sources of Psychological Support for East Asians Reporting a Low Level of Mental Health}

Mental illness affects individuals of all backgrounds, regardless of racial, ethnic, cultural, gender, or social boundaries (Vigo, Thornicroft, \& Atun, 2016). East Asian countries are not immune from this global phenomenon of mental illness. Hundreds of millions of East Asians suffer from mental illness, and despite the great need for treatment for this everincreasing population, professional mental health workers and treatment providers are scarce (Hossain, 2006). Evidence shows that in addition to seeking help from professionals, people suffering from low mental health rely on different supports sources, such as family (Hanzawa et al., 2009; Lam et al., 2010) and alternative medicines (Eguchi, 1991; Hanzawa et al., 2009; Hohenshil et al., 2015; Kleinman, 1980; Thirthalli et al., 2016). Studies also show that East Asians tend to use alternative medicines for treatment of low mental health (Thirthalli et al., 2016). Despite the importance of these resources for East Asians suffering from mental illness, there is a paucity of research on their prevalence in this particular population. Moreover, we know little about how these support sources differ by country. The purpose of the present study is to examine where East Asians seek assistance for low mental health and how these help-seeking behavior patterns differ across four East Asian countries.

Mental illness is prevalent in East Asian countries (Cabinet office, 2008; Kessler et al., 2005; Lim et al., 2010; Tsai \& Cho, 2011). According to the 2008 Annual Report on Government Measures for Persons with Disabilities, there were over 3 million individuals who reported to be suffering from mental disorders in Japan (Cabinet office, 2008). In neighboring China, 100 million individuals were reported to suffer from mental illness (Lim, Lim, Michael, Cai, \& Schock, 2010). Taiwan's prevalence rate for common mental disorder was $23.8 \%$ in 2010 (Fu, Lee, Gunnell, Lee, \& Cheng, 2013). In South Korea, the lifetime rate was 33.5\% (Kessler et al., 2005). Despite such prevalence, professional treatment for mental illness in East Asian nations is still considered to be in the early stages of development. Merely applying Western mental health knowledge and treatment to East Asian patients is not sufficient (Au, 2017); it is critical to understand the cultural background of each individual East Asian nation in order to develop effective, culturally based treatment modalities. 
It is common among East Asians who suffer from mental illness to seek, in addition to professional treatment (if it is utilized and/or accessible), alternative medicine and support from friends and family (Eguchi, 1991; Kleinman, 1980; Thirthalli et al., 2016). A more in-depth study on prevalence rates of different types of psychological support would contribute to treatment development in the future (Eguchi, 1991; Hanzawa et al., 2009; Kleinman, 1980; Lam et al., 2010; Thirthalli et al., 2016).

In the review of the literature on mental illness and usage of support resources among East Asian cultures, the following four topics are the most prominent. A majority of the literature is about the prevalence of mental illness in East Asian cultures (e.g., anxiety disorders, mood disorders, psychotic disorders). Second is literature on professional psychological support for persons with such disorders. Third is information on familial support and stigma regarding mental health problems. Finally, traditional and alternative medicinal practices for persons with mental health problems are addressed. After these areas of the literature are examined, the research questions that guided this study are presented.

\section{Prevalence of Mental Illness In East Asian Cultures}

Lim, Lim, Michael, Cai, and Schock (2010) discussed mental illness as the most widespread disease in China, with an estimation of 100 million Chinese citizens, which is about $7.5 \%$ of the Chinese population suffering from various psychological disorders. The highly competitive educational system and business environment, along with the rapid urbanization of the rural Chinese population, make mental health worthy of both local and global attention (Hohenshil, Amundson, \& Niles, 2015). Similarly, Japan also has a continuously increasing need for mental health treatment availability. According to a study by the Japanese government (Cabinet Office, 2008), there are an estimated 3 million Japanese citizens, which is about $2.3 \%$ of the population suffering from mental health disorders.

Several recent studies have demonstrated that the Japanese family unit has experienced increasing divorce rates, along with child abuse and domestic violence, increasing significantly (Iwasaki, 2005). Bullying and violence among teenagers has become a more severe issue (Iwakabe, 2008), and suicide rates have increased significantly since 1998, with over 30,000 suicides every year since the late 1990s (National Police Agency, 2009). 
These mounting social and mental issues demonstrate the need for implementation of effective mental health interventions and treatments among these populations.

Neighboring Taiwan also demonstrates a great need for mental health services. The prevalence of probable common mental disorders rose from $11.5 \%$ in 1990 to $23.8 \%$ in 2010 (Fu, Lee, Gunnell, Lee, \& Cheng, 2013). From 1976 to 2009, the suicide rate increased to as high as 19.30 per 100,000 persons (Tsai \& Cho, 2011). Societal issues such as transnational marriage (Shu, Lung, \& Chen, 2011) have raised significant mental health issues for families and individuals in Taiwan. In South Korea, the lifetime and 12-month prevalence for all types of DSM-IV disorders were 33.5\% and 20.6\%, respectively. Among the four East Asian countries that are the focus of the current study, the prevalence of psychiatric disorders in South Korea was the highest (Kessler et al., 2005). Despite the urgent need for mental health treatment as a result of increasing need in East Asian countries, knowledge of the prevalence of societal support for persons with mental disorders is lacking.

\section{Current Societal Psychological Support for Persons with Such Disorders}

China currently has some existing sources of psychological support. Hou and Zhang (2007) identified three such sources: psychiatric hospitals, school counselors, and private practitioners. The psychiatric hospitals mainly focus on severely ill patients, with medications prescribed by physicians. In school settings, many school counselors are also homeroom teachers who hold psychological-counseling responsibilities (Hohenshil et al., 2015). However, such school counselors are scarce, with only $10 \%$ employed in urban schools and less than 1\% employed in the rural provinces (Jiang, 2007, as cited in Cook, Lei, \& Chiang, 2010). There are other counselors who work for the private sector, most of whom are located in larger coastal cities (Hohenshil et al., 2015).

Compared to China, the counseling profession in South Korea is more developed, with greater availability of mental health professionals. School counseling was first introduced to South Korea in the 1950s (Hohenshil et al., 2015), full-time counselors are currently employed in 2,045 out of 11,170 schools (Hohenshil et al., 2015). Out of 178 school boards, 124 
of them built their own school-counseling centers with on-site psychologists, registered school counselors, and social workers (Lee et al., 2012). Other non-school counseling resources available to the public include university counseling centers and community counseling centers (Hohenshil et al., 2015).

By contrast, Taiwan's overall mental health system is still in its infancy. While guidance counseling in school systems was first introduced to Taiwan more than 60 years ago, during the 1950s (Chen, 1999), the availability of guidance counselors remains scarce. A survey indicated that one school counselor is responsible for 20 or more classes (Ministry of Education, 2011). Besides school counselors, community-related mental health counselors are beginning to be more readily available to the public (Lin, 2000).

As in other East Asian countries, the availability of counseling services in Japan remains insufficient, but the number is steadily growing. In 2011, a total of 24,660 certified clinical psychologists worked in various settings, such as hospitals, schools, private clinics, universities, and companies (Foundation of the Japanese Certification Board for Clinical Psychologists, n.d.). The total number of school counselors has also increased. In 2006, there were school counselors in 10,158 schools, which was a significant increase from 154 schools in 1995 (Hohenshil et al., 2015). While societal counseling support might not be widely available to individuals in East Asian countries for various reasons, familial assistance seems to serve as a major source of support to persons with mental illness.

\section{Familial Support and Stigma for Persons with Such Disorders}

The need of familial assistance in East Asian countries can be understood from the role of stigma for persons with mental health disorders. Fabrega (1991) argued that individuals in Chinese society suffering from mental illness carry extreme shame, as they blame themselves for their illness. The families of individuals who suffer from mental illness also experience much shame (Jenni, 1999). This seems to be true among individuals from both the Chinese mainland and its neighbor Taiwan (Mellor et al., 2012). Similar to Chinese society, individuals and families with mental illness in Japan and Korea also demonstrate feelings of 
embarrassment and shame (Hanzawa et al., 2009). As a result, some individuals with mental illness and their caregivers tend to choose familial assistance over outside support and attempt to keep the illness a family secret (Hanzawa et al., 2009; Lam et al., 2010). While societal resources are scarce, and mental illness carries such enormous shame and stigma among individuals from East Asian countries, many turn to traditional and alternative medicine for assistance.

\section{Traditional and Alternative Medicine Practices For Mental Health Issues}

It is common practice for East Asians with different health conditions to seek alternative medicine. Up to $80 \%$ of Chinese individuals with various health conditions consult practitioners of traditional, alternative, and complementary medicine at some point (Thirthalli et al. 2016). Many individuals with mental illness consider traditional, alternative, and complementary medicine in addition to biomedicine provider (Kleinman, 1980). Some of these alternative-medicine strategies include traditional medicine such as acupuncture, moxibustion, massage therapy, qigong, tai chi, and folk therapy (Thirthalli et al., 2016). Similar practices and beliefs are shared by individuals in Japan (Eguchi, 1991), Korea (Hohenshil et al., 2015), and Taiwan Hohenshil et al., 2015).

Despite cultural similarities among Asians, research suggests that there might be significant differences among Asian subgroups in attitudes toward mental health issues (Fung \& Wong, 2007). One study found that Korean immigrants were less likely to endorse marital violence than their Chinese counterparts (Yoshioka, DiNoia, \& Ullah, 2001). Another study suggested that Koreans are more likely to endorse "traditional, non-Western beliefs, including both 'non-Western physiological' and supernatural beliefs'" compared to Chinese (Fung \& Wong, 2007).

With all the above sources of assistance, we know little about the prevalence of usage by individuals with low mental health. There is also lack of quantitative analysis studies on how East Asian countries differ from each other in assistance usage. The present study addresses this gap in the literature. 
The present study was guided by eight research questions about persons reporting a low level of mental health: 1) What is the usage rate of kin as a support resource? 2). Are there statistically significant differences in the use of kin as a support resource by country? 3). What is the usage rate of non-kin as a support resource? 4). Are there statistically significant differences in the use of non-kin as a support resource by country? 5). What is the usage rate of alternative medicine as a support resource? 6). Are there statistically significant differences in the use of alternative medicine as support resource by country? 7). What is the usage rate of professional mental health services as a support resource? 8). Are there statistically significant differences in the use of professional mental health services as support resource by country?

\section{Method}

\section{Design}

This study employed a retrospective, cross-sectional observational analysis in order to determine between-country differences in the utilization of different types of health assistance and the prevalence of East Asians' reporting a low level of mental health. Data were obtained from the East Asian Social Survey (EASS) (Iwai, Li, Kim, \& Chang, 2010). EASS is a biennial, cross-national social survey project of the following four types of General Social Surveys in East Asia: (1) the Chinese General Social Survey (CGSS); (2) the Japanese General Social Survey (JGSS); (3) the Korean General Social Survey (KGSS); and (4) the Taiwan Social Change Survey (TSCS). The purpose of these surveys is to compare and contrast the varieties of social life in these geographic areas to produce and disseminate academic survey data sets in East Asia. The information derived from the surveys includes general human health issues, such as specific illnesses or diseases, physical functioning, caretaking and assistance received from family members or friends when needed, and lifestyle choices. EASS utilized multistage sampling procedures to gather data: China, three-stage PPS; Japan, two-stage stratified random sampling (stratified by regional block and population size); Korea, multistage area probability sampling; Taiwan, three-stage stratified PPS sampling (PSU [township], village, and individual person). 
Although EASS assessed a number of variables, the current dissertation focuses on mental health and different sources of assistance. The specific variables of the study were level of mental health (continuous), use of kin (ordinal), use of non-kin (ordinal), use of alternative medicines (ordinal), use of mental health professionals (ordinal), and country (nominal).

\section{Participants}

The demographics for participants by country were as follows. The initial sample size of the 2010 Chinese General Social Survey was 5,370, with 3,866 responding; the sample was composed of Chinese aged 18 and above. The initial sample size of the 2010 Japanese General Social Surveys was 4,500, with 2,496 responding; the sample was composed of men and women aged 20-89 living in Japan. The initial sample size of the 2010 Korean General Social Survey was 2,500, with 1,576 responding; the sample was composed of adult citizens aged 18 and over who lived in South Korean households. The initial sample size of the 2011 Taiwan Social Change Survey 2011 was 4,424, with 2,199 responding; the sample was composed of population registers.

A total of 3,451 respondents who identified as low mental health were selected in which consisted of a total 1,495 males and 1,956 females. The majority of the sample was from the age 20 to 59 which consisted about $71.2 \%$ of the total sample. In addition, $71 \%$ was reported married while $15.6 \%$ reported never married. The range for the number of years of education was 21 years with a mean of 10.14 years.

\section{Measures}

All data were drawn from measures contained in the East Asian Social Survey (EASS; Iwai et al., 2010). The item numbers that appear in this subsection refer to the item numbers in the data codebook and files of EASS.

Mental health. Mental health was assessed using the Short-Form Health Survey, Version 2, Mental Health subscale (sf12_mh). The Medical Outcomes Study's Short-Form 12-Item Health Survey (SF-12) is a briefer form of the Medical Outcomes Study's 36-Item Short-Form Health Survey 
(SF-36), which assesses the general health, physical functioning, social functioning, physical role, emotional role, mental health, vitality, and bodily pain (Salyers, Bosworth, Swanson, Lamb-Pagone, and Osher, 2000; Ware, Kosinski, \& Keller, 1996). Previous studies suggested that the SF-12 is a reliable and valid instrument for assessing overall mental and physical health in the general population and of people with severe mental illness (Salyers et al., 2000).

The Mental Health (MH) subscale of the SF12 is composed of two items: "SF_Q11. Have you felt downhearted and depressed during the past 4 weeks?" and "SF_Q12. During the past 4 weeks, how much of the time has your physical health or emotional problems interfered with your social activities (like visiting friends, relatives, etc.)" The response options for each question were "(a) All of the time $=1$," "(b) Most of the time $=2$, , "(c) Some of the time $=3$," "(d) A little of the time $=4$," "(e) None of the time $=5$," "(f) Not asked $=7$," and "(f) DK, refused $=8$." Subscale scores can range from 0 to 100 , with 100 representing the most favorable state. A low level of mental health was operationalized by use of a median split.

Use of kin as a support resource. The use of kin as a support resource was assessed using a single-item: "During the past 12 months, did your kin (i.e., family or relatives) do the following things for you when you needed it? If yes, how often?" The possible responses to this question were "Very often (1)," "Often (2)," "Sometimes (3)," "Seldom (4)," "Not at all (5)," "No, do not have such needs (6)," "No such persons available (7)," "Not asked (77)," and "Don't know/Refused (88)." Because the question specifically asked if the respondents received support when they needed it, response number 6 is not useful as an indicator of frequency of support. Similarly, because the question is whether kin provided support "no such persons available" implies that the person does not have kin, so "no such persons available" is not a useful indicator of frequency of support. Thus, responses 6 and 7 were excluded. In addition, scores of 77 and 88 were excluded from all analyses (i.e., Japan). The item was recoded so that a high score meant higher rate of support

Use of non-kin as a support resource. The use of non-kin as a support resource was assessed using a single item: "During the past 12 months, did your non-kin (friends, colleagues, or neighbors) do the following things for 
you when you needed it? If yes, how often?" The possible responses to this question were "Very often (1)," "Often (2)," "Sometimes (3)," "Seldom (4)," "Not at all (5)," "No, do not have such needs (6)," "No such persons available (7)," "Not asked (77)," and "Don't know/Refused (88)." Because the question specifically asked if the respondents received support when they needed it, response number 6 is not useful as an indicator of frequency of support. Similarly, because the question is whether non- kin provided support "no such persons available" implies that the person does not have a person described as such, so "no such persons available" is not a useful indicator of frequency of support. Thus, responses 6 and 7 were excluded. In addition, the score of 77 was excluded from all analyses (i.e., Japan). The item was recoded so that a high score signified a higher frequency of support.

Use of mental health professionals. The use of mental health professionals as a support resource was assessed using a single item (v55): "During the past 12 months, did your professional workers (e.g., social workers, caretakers, or therapists) do the following things for you when you needed it? If yes, how often?" The possible responses to this question were "Very often (1)," "Often (2)," "Sometimes (3)," "Seldom (4)," "Not at all (5)," "No, do not have such needs (6)," "No such persons available (7)," "Not asked (77)," and "Don’t know/Refused (88)." Similar to the above two measures, responses 6 and 7 were excluded. In addition, the score of 77 was excluded from all analyses (i.e., Japan). The item was recoded so that a high score signified higher rate of support.

Use of alternative medicine. The use of alternative medicine as a support resource was assessed using three items (v46, v47, and v48): "Have you ever received the following treatment during the last 12 months?" The three categories of alternative medicines were "(a) Acupuncture or moxibustion (cupping)" (v46), "(b) Oriental herbal medicine (v47)," and "(c) Acupressure or clinical massage" (v48). The possible responses to each item were "Yes (1)" or "No (2)." "Yes" responses were recoded into "1" and "No" responses were recoded into " 0 ". Answers with no on any of the items were recoded as " 0 " and answers with yes on any one or more of the items were recoded as " 1 ". Note that cupping is not included in JGSS due to its lack of popularity in Japan. 
Country. The country/region of respondents, China, Japan, South Korea, and Taiwan, were respectively coded as "1," "2," "3," and "4."

Data Analysis. First, data screening and cleaning including detection of anomalies, outliers, and missing value patterns were conducted. Second, Listwise deletion was used to treat missing cases or observations. For Research Questions 1, 3, 5, and 7 (rates), frequencies and percentages were calculated. For Research Questions 2, 4, and 8, ordinal variables involved in the study could be treated as interval variables given their specific Likert structure and the large sample size (Jamieson, 2004). As such, differences between countries were analyzed using a one-way ANOVA. To fully understand differences among countries, post hoc tests were conducted. The Chi-square test was used to address Research Question 6, where the dependent variable is alternative medicine, and the independent variable is country.

\section{Results}

Although the overall EASS survey included 10,137 respondents across the four East Asian countries, the Taiwan sample was not asked questions regarding mental health. Thus, the analytic sample of this study included 3,451 respondents (42.4\% Chinese, 32.9\% Japanese, and 27.7\% South Korean) who scored below the median on the SF-12 mental health subscale. Prior to conducting the primary analysis, the data were screened for missing values and outliers on the study variables as well as violations of specific statistical assumptions. Analysis of missing value patterns indicated that missing values were well below $1.2 \%$ for the alternative medicine types. The Japanese sample was not asked the question on social support sources. Thus, the Japanese sample did not have data on kin, non-kin and professional mental health services. For the remaining two countries, there were $8.8 \%$ missing data for non-kin support, $9.1 \%$ for kin support and $57.2 \%$ for professional mental health services. Examination for outliers revealed that four participants had scores above 3 standard deviations from the mean for non-kin support and six participants had a score above 3 standard deviations for professional support. In this study, complete case analysis was used without having to exclude outliers. Although one-way ANOVA is robust to assumptions of normality, in the current study, the author tested for this assumption using skewness and kurtosis values for each of the 
continuous variables. Results of the analysis showed that in absolute value terms, the skewness values ranged from $.24(\mathrm{SE}=.08)$ for kin support to $1.88(\mathrm{SE}=.08)$ for professional support. Similarly, again in absolute value terms, the kurtosis values ranged from $.62(\mathrm{SE}=.16)$ for kin support to $3.24(\mathrm{SE}=.18)$ for professional support. The range of values for the two statistics indicate that the continuous variables appear to range from normally distributed to relatively skewed.

The first research question sought to determine the usage rate of kin as a support resource among East Asians reporting low mental health. To address this research question, we calculated descriptive statistics of kinsupport as indicated by the respondents. Table 2 presents how often the respondents' kin extended emotional support to respondents. The majority (75.6\%) of East Asians reporting low mental health received emotional support from their kin at least sometimes in the past 12 months. Indeed, about $42 \%$ of the participants reported having received emotional support from their kin often or very often.

The second research question was concerned with whether there were statistically significant differences in the use of kin as a support resource by country. A one-way ANOVA was conducted to compare if mean frequency of reported kin emotional support differed by country. The results indicated that there was statistically significant difference between countries in the use of kin as a support resource, $F(1,2103)=83.149, p=.000)$. The Chinese respondents reported higher use of kin emotional support $(M=$ $3.37, S D=1.02)$ than South Korean respondents $(M=2.92, S D=1.19)$.

Similar to the first research question, the third research question sought to determine the prevalence of non-kin as a support resource among East Asians reporting low mental health. Again, the descriptive statistics were calculated for non-kin support as reported by the respondents. Table 1 presents how often respondents received emotional support from non-kin. The majority (67\%) of East Asians reporting low mental health received emotional support from non-kin at least sometimes in the past 12 months. About 27\% reported having received emotional support from such source often or very often. 
The fourth research question aimed at determining whether there are statistically significant differences in the use of non-kin as a support

resource by country. A one-way ANOVA was conducted to compare if mean frequency of non-kin support differed by country. The results indicated that there was statistically significant difference between countries in the use of non-kin as a support resource, $F(1,2103)=6.51, p=.01)$. The South Korean respondents reported higher use of non-kin support $(M=2.92, S D$ $=1.14)$ than Chinese respondents $(M=2.79, S D=1.02)$.

The fifth research question sought to determine the usage rate of alternative medicine as support resource by East Asians reporting low mental health. We calculated percentages of respondents who reported using three types of alternative medicine: Acupuncture/Moxibustion, Oriental herbal medicine, and Acupressure/Clinical massage. Table 2 presents the number and percentage of East Asians reporting low mental health who used alternative medicine in the past 12 months. One quarter (25\%) of East Asians reporting low mental health used herbal medicine in the past 12 months, while about $16 \%$ used acupressure, and 17\% used acupuncture.

In addition to determining the usage rate of alternative medicine as support resource by East Asians reporting low mental health, in the sixth research question, we sought to determine whether there are statistically significant differences in the use of alternative medicine as support resource by country. Three chi-square tests of independence were calculated: one for each alternative medicine type. Table 2 presents cross tabulations and chisquare tests for alternative medicine by country. As can be seen from the table, there are significant differences in the use of acupuncture among the three countries, $X^{2}(2,3)=270.33, p=.00$. Acupuncture seems to be most common type of alternative medicine in South Korea with $34 \%$ of the South Korean participants reporting its use in the past 12 months. The table also shows that there are significant differences in the use of herbal medicine among the three countries, $X^{2}(2,3)=270.34, p=.00$. Herbal medicine seems to be the most common type of alternative medicine in China with $38 \%$ of the Chinese participants reporting its use in the past 12 months. Finally, there are significant differences in the use of acupressure among the three countries, $X^{2}(2,3)=82.48, p=.00$. Acupressure seems to be the most common type of alternative medicine in Japan with about $23 \%$ of the participants reporting its use in the past 12 months. 
The seventh research question sought to determine the usage rate of professional mental health services as a support resource. We calculated descriptive statistics of the use of professional mental health services as indicated by the respondents. Table 1 presents how often the respondents' received professional mental health services. The majority (70\%) of East Asians reporting low mental health did not receive professional mental health services during the last 12 months. Indeed, only about $14 \%$ reported having used professional mental health services as a support resource at least sometimes.

The last research question was concerned with whether there are statistically significant differences in the use of professional mental health services as support resource by country. A one-way ANOVA was conducted to compare if mean frequency of reported use of professional mental health services differed by country. The results indicated that there was a statistically significant difference between countries in the use of professional mental health services as a support resource, $F(1,2103)=$ $18.47, p=.00)$. The Chinese respondents reported higher use of professional mental health services $(M=1.60, S D=.90)$ than South Korean respondents $(M=1.36, S D=.83)$.

\section{Discussion}

This study examined support preferences for mental health care assistance among East Asians reporting low mental health. The study examined whether there are differences between countries in rates of usage of support sources. More specifically, this study addressed the following research questions. What is the usage rate of kin as a support resource? Are there statistically significant differences in the use of kin as a support resource in each country? What is the usage rate of non-kin as a support resource? Are there statistically significant differences in the use of non-kin as a support resource in each country? What is the usage rate of alternative medicine as a support resource? Are there statistically significant differences in the use of alternative medicine as support resource in each country? What is the usage rate of professional mental health services as a support resource? Are there statistically significant differences in the uses of professional mental health services as support resource in each country? 
Next, results are presented and discussed. Toward the end of the section, limitations and implications of the findings are presented.

The first research question sought to determine the usage rate of kin as a support resource among those reporting low mental health. The findings indicated that among East Asians reporting low mental health about $76 \%$ reported having received emotional support for their mental health problems. Given what we know from the literature on mental health, this prevalence rate seems to confirm the findings from previous studies. One reason for this high level of the usage of kin as a support resource among East Asians is that individuals suffering from mental illness carry a tremendous sense of shame in the East Asian societies and exposing such shame to outside of family is socially unacceptable (Fabrega, 1991; Hanzawa et al., 2009; Jenni, 1999; Mellor et al., 2012). Losing face due to mental illness not only reflects negatively on the individual, but also on families and their ancestors (King \& Bond, 1985). Comparison of this finding with that of other studies confirms that East Asians tend to rely on kin support for mental health problems. For instance, it was found in studies that East Asians with mental illness and their caregivers would tend to choose familial assistance over outside support to keep the illness a family secret (Hanzawa et al., 2009; Lam et al., 2010). Another possible reason may be due to the scarcity of professional assistance. Indeed, previous studies have found that the ratio of Chinese professional counselors to patients is about 2.4 per 1 million (Chinese Psychological Society, 2004) compared to 3,000 counselors per 1 million people in the US (Hohenshil, Amundson, \& Niles, 2015). In Japan, more than half of certified clinical psychologists earn less than the national average and $46.1 \%$ work only part- time (Hohenshil, Amundson, \& Niles, 2015). With the lack of professional assistance, individuals with low mental health have no choice but to rely on the more accessible familial assistance. While both explanations contributed to the findings, the cultural reason is the most influential one. Studies have shown that East Asians are deeply influenced by Confucian philosophy which views disabilities in terms of fate and one should accept one's role handed down by destiny (Lam et al., 2010). Furthermore, as collective societies, having a family member with mental illness is seen as loss of face for the family (Lam et al., 2010). As a result, many are reluctant to both publicly state that their relatives are afflicted with mental illness and to seek assistance outside of the family. 
Concerning the second research question which was concerned with whether there are significant differences in the use of kin as a support resource by country, the results indicated that there was a statistically significant difference between the two East Asian countries of China and South Korea. More specifically, the Chinese respondents reported higher use of kin emotional support than South Korean respondents. One possible explanation for this finding is the relatively more accessibility to professional mental health assistance in South Korea compared to China. Currently, 2,045 out of 11,740 schools in South Korea have onsite full-time school counselors, registered or unregistered (Lee, Suh, Yang, \& Jang, 2012). That is about $17 \%$ compared to $10 \%$ of urban Chinese schools staffed with mental health educators whose job descriptions are primarily dealing with administrative aspects of students' education and development (Hohenshil, Amundson, \& Niles, 2015). However, this explanation conflicts with this study's finding on professional mental health assistance usage which will be discussed later. Another plausible explanation is that South Korean citizens have higher accessibility to religious support systems than their counterparts in China. Previous studies have suggested that religious social support and church attendance might reduce mental health issues (Nooney \& Woodrum, 2002; Schwadel \& Falci, 2012). Compared to China, South Korea has experienced a tremendous increase in its religious population. The Protestant population in South Korea has increased from 2.5\% to 19.7\% between 1960 and 1995 (Hong, 1999). The accessibility of religious social support is abundant compared to neighboring China. It is estimated that China has only about a $3 \%$ Protestant population because most belong to underground churches without proper resources and support (Fiedler, 2010). Moreover, a study showed that Protestantism in South Korea has a negative association with suicide rate and stress level (Jung \& Olson, 2014). Such accessible and useful religious social support in South Korea might have contributed to the reduction of needs of kin support.

Between these two explanations, the most probable is the widely accessible non-kin religious assistance in South Korea. Various studies have shown that South Koreans have greatly increased their religious participation (Kim, 2003; Lee 2009), with much of their religious activities involving spiritual support and assistance (Lee, 2009). Such a high level of organized non-kin support is not available or accessible to individuals in China where religious activities are largely restricted (Fiedler, 2010). 
Turning to the third research question which focused on the usage rate of non-kin support, the findings showed that the majority $(67 \%)$ of East Asians reporting low mental health received emotional support from non-kin at least sometimes in the past 12 months. This finding is consistent with previous studies where it was found that individuals with mental health issues prefer seeking help from informal sources such as family and friends (Na, Ryder, \& Kirmayer, 2016; Picco et al., 2016). One study conducted in Hong Kong supported this finding. Out of 10,179 adult respondents, $46.5 \%$ of the participants preferred using friends and family as assistance for depression symptoms, $24.9 \%$ preferred psychiatrist, $22.8 \%$ preferred psychologist, and $19.9 \%$ chose general practitioner (Chin, Chan, Lam, Lam, \& Wan, 2015). While $67.6 \%$ of the participants indicated that they prefer receiving help from professionals such as psychiatrists, psychologists, and general practitioners when asked, only $24.3 \%$ of the participants which have screened-positive with depression reported receiving professional mental health services over a period of 12 months. One plausible explanation for such a finding is the high accessibility to non-kin assistance. Another plausible explanation of the higher rates of non-kin assistance seeking behavior is that it is a more socially acceptable assistance than receiving professional help. For instance, it is a norm in Chinese culture that one expects help within one's social network, and it would be uncomfortable for an individual considering resources from outsiders (Tata \& Leong, 1994). This mentality influences help-seeking behavior in which non-kin assistance might be a better choice (Tata \& Leong, 1994). The latter offers a stronger explanation since previous studies have also suggested such help-seeking behavior.

Similar to research question 2 , the fourth research question sought to determine if countries differ in the use of non-kin as a support resource. The results indicated that there was statistically significant difference between countries in the use of non-kin as a support resource such that South Korean respondents reported higher use of non-kin support than Chinese respondents. No prior studies were identified that compared the prevalence of Chinese and South Korean individuals with low mental health using nonkin assistance. However, one study indicated that while Chinese respondents expect help within their social network (Tata \& Leong, 1994), they showed concern about sharing their thoughts and problems with friends as this could affect their relationships negatively (Diego, 2016). 
Other studies suggested that Chinese culture focuses on the repression of feelings, rather than focusing on the expression of feelings or emotional vulnerability (Ots, 1990; Parker, Gladstone \& Chee, 2001). In contrast, a study on Korean American's help-seeking behaviors indicated that most tend to seek advice about their mental health problems from non-mental health professionals such as friends and religious healers (Cheung, Leung, \& Cheung 2011). These studies seemed to suggest the significant difference between Chinese and South Korean participants on the prevalence of utilizing non-kin as a resource is embedded in the culture. It is more acceptable with less cultural hindrance in the Korean culture for individuals with mental health issues to express their concerns and seek for assistance from non-kin. Another plausible explanation is the availability of social support from religious organizations in South Korea. Approximately onefourth of South Korean self-identified as Protestants (Hong, 1999). Further, South Korean Protestantism is known for a high level of religious attendance where it is not unusual for a church to offer multiple events and services weekly (Jung \& Olson, 2014). With such a wide availability of social support, South Koreans would be more likely to receive non-kin religious support than their Chinese counterparts where religious activities are largely restricted (Zhang \& Zhu, 2011). The most probable explanation that both of the aforementioned reasons equally contributed to the finding.

Concerning the fifth research question that sought to determine the usage rate of alternative medicine as support resource by East Asians reporting low mental health, the findings indicated that $25 \%$ of respondents reported using herbal medicine; about 17\% used acupuncture whereas $16 \%$ reported using acupressure. This usage rate is much higher than the rates reported in studies from Western countries. According to a U.S. national survey conducted in the year of 2000, of the 9,566 respondents, 1,576 reported the use of alternative medicine in the past 12 months which is about $14.5 \%$ of the total sample (Unützer et al., 2000). Among those who reported using alternative medicine in the past 12 months, only about $15.0 \%$ reported using alternative medicine to treat mental or emotional problems (Unützer et al., 2000). The definition of alternative medicine on this particular survey included homeopathic medicine, acupuncture, massage therapy, herbal medicine, and spiritual healing (Unützer et al., 2000). 
Although usage rates for acupuncture and acupressure are not as high as that of herbal medicine, acupuncture and acupressure are considerably common among East Asians reporting low mental health compared to the U.S. For instance, studies found that only about $6.3 \%$ of Americans used acupuncture as a conjoint treatment for medical conditions (Zhang, Lao, Chen, \& Ceballos, 2012). While acupressure has recently gained some acceptance by in the Western world as a valid means for treating symptoms of illness, its usage rate in the U.S. is yet to be studied (Hakverdioglu \& Turk, 2006). The usage rate for mental health purposes is assumed to be much lower. One explanation for the high rate of using alternative medicine for treating mental health issues among East Asians is that herbal medicine, acupuncture, and acupressure have been practiced in East Asia for more than 2000 years (Hakverdioglu \& Turk, 2006; Leslie, 1976). The trust in alternative medicine to treat health issues is much higher for East Asians than for individuals in the Western world. Another plausible explanation is that East Asian countries have institutionalized alternative medicines into the healthcare system which greatly increased the accessibility (Shim, 2016). For instance, traditional Chinese medicine doctors are granted the right to practice biomedical tests and treatments as well as alternative treatments in China (Xu \& Yang, 2009). While South Korea has a different healthcare system where alternative medicine doctors and biomedicine doctors are licensed and practiced under separate systems, both China and South Korea grant alternative medicine a legal recognition and greater accessibility than the Western societies. Consequently, compared to Western world, East Asians have a higher usage rate of alternative medicine. Both the credibility of alternative medicine and its accessibility contributed to its high usage rate in East Asia.

The sixth research question addressed the topic do East Asian countries differ in the usage rate of alternative medicine. The results showed that there are indeed significant differences in the use of alternative medicine among the three countries. More specifically, the results indicated that acupuncture seems to be the most common type of alternative medicine in South Korea whereas herbal medicine and acupressure, respectively, appear to be the most common type of alternative medicine in China and Japan. While there are previous cross-national studies comparing the three countries on traditional East Asian medicine (Shim \& Kim, 2018), the present research was the first study comparing the three 
countries' alternative medicine use as a treatment for individuals with low mental health. One plausible explanation for herbal medicine is the most common alternative medicines used in China compared to Japan and South Korea is that herbal medicine is more accessible than Japan and South Korea. In the Chinese healthcare system, biomedical doctors are allowed to practice traditional Eastern alternative medicine (Shim, 2016). Likewise, traditional East Asian medicine doctors can practice biomedicine (Shim, 2016). In contrary, the South Korean healthcare system has more restricted practice rights where biomedical doctors and traditional East Asian medicine doctors are exclusively separated on their scope of practice (Shim, 2016). Furthermore, Japan has no professional category of traditional East Asian medicine that are comparable to biomedical doctors, and there are no medical schools designated for traditional East Asian medicine. The accessibility to non-biomedical treatment is relatively limited compared to other East Asian countries. Consequently, individuals in China have higher accessibility to herbal medicine than individuals of neighboring countries.

Another plausible explanation is that most herbal medicine is not covered by South Korean government insurance which limited the accessibility to many patients (Kim, Kim, Noh, Kang, Kim, Yang, \& Lee, 2012). On the other hand, the South Korean government has increased its support of promoting traditional Korean medicine since 2008 in which acupuncture has grown as the most popular type traditional medicine (Woo, Park, Lee, Ahn, Kwon, \& Koo, 2014). One explanation for South Korean having the highest acupuncture usage rate is the increased availability and affordability of acupuncture. Treatments including dry cupping, moxibustion, and acupuncture are fully covered by the South Korean government insurance (Kim, Kim, Noh, Kang, Kim, Yang, \& Lee, 2012) whereas an average acupuncture treatment fee in China was 19.32 CNY (2.8 USD) (Zhao, 2011).

While herbal medicine and acupuncture are popular in China and South Korea, Japan has a significantly lower usage rate of these two types of traditional medicine but has a significant higher usage rate of acupressure among individuals reported low mental health. One plausible explanation is the lack of general trust of traditional medicine. The Japanese government does not grant a license to traditional medicine practitioners (Shim 2016). In Japan, there are only vocational schools for Eastern medicine technicians, 
such as acupuncturists and acupressure therapists (Shim, 2016). In addition, doctors are discouraged from using traditional medicine as conjoint treatment with biomedicine (Shim, 2016). Therefore, the accessibility of traditional medicine is significantly limited compared to its neighboring countries. However, one explanation for Japan having the highest usage rate of acupressure is its nonintrusive nature. While both herbal medicine and acupuncture require an individual to consume medicine and muscle penetration by needles, acupressure requires only placing pressure on the surface of the body (Hakverdioglu \& Turk, 2006). Another potential explanation is its similarities to the traditional Japanese treatment called Shiatsu. Shiatsu can be dated back to the 14th century and was popularized in the 17th century. The Shiatsu techniques are based on traditional Chinese medicine which explanations its similarity to acupressure. This researcher's belief that all of the above explanations contributed to the findings.

The seventh research question sought to determine the usage rate of professional mental health services as a support resource. Only about $14 \%$ of the respondents reported having used professional mental health services as a support resource at least sometimes. Previous studies on the use of professional mental health services in East Asia indicate similar findings (Park et al., 2012; Xiang, Yu, Sartorius, Ungvari, \& Chiu, 2012). One plausible explanation of such finding is the scarcity of qualified, trained mental health professional assistance. For instance, China has approximately 15,000 trained psychiatrists to serve a population of 1.3 billion (Ma, 2011). In South Korea, there is still no licensure system for counselors. Another plausible explanation is the stigma of mental illness which brings shame not only to the individual but also the family (Fabrega, 1991; Hanzawa et al., 2009; Jenni, 1999; Schomerus et al., 2012). Such stigma prevents those who are in need to seek outside assistance. As a result, individuals with mental illness tend to choose assistance within their families or social circle instead of professionals (Hanzawa et al., 2009; Lam et al., 2010). In addition to the above reasons, another explanation would be the emphasis of familial social support in the East Asian culture in which decreases the need for professional assistance. One study found that higher social support was associated with decreased perceived need for mental health assistance (Arnault, Gang, \& Woo, 2018). Another plausible explanation might be affordability. Patients cannot afford to see a mental 
health professional even if there is one available especially in rural area (Ma, 2011). While all of the above explanations played a role to the finding, previous studies have shown that the major contributors are the paucity of professional mental health assistance and the stigma of mental illness (Fabrega, 1991; Hanzawa et al., 2009; Jenni, 1999; Lam et al., 2010; Ma, 2011; Schomerus et al., 2012).

The findings for the final question indicated that there was a statistically significant difference between China and South Korea in the use of professional mental health services as a support resource with China being higher. This finding needs to be interpreted in light of the fact the level of the reported professional mental health support is generally low across the two countries. This difference between the two countries can be explained by again the higher accessibility to non-kin religious social support in South Korea in which reduce the self-perceived need for professional assistance (Arnault, Gang, \& Woo, 2018). Another plausible explanation lies in the Chinese healthcare system. As mentioned above, the Chinese healthcare system allows biomedical doctors to practice traditional Eastern alternative medicine while the South Korean defined the two practices exclusively (Shim, 2016). As a result, Chinese respondents who seek traditional medical treatment would also receive treatment from mental health professionals such as psychiatrists. The most plausible explanation is the high level of accessibility to non-kin social support in South Korea which reduce the help-seeking behavior for mental health professionals. It seemed to be in line with a recent study conducted on South Korean women (Arnault, Gang, \& Woo, 2018).

Overall there are several limitations to this research. The major limitation of this study is that, while the data shows an association between resources types among individuals reported with low mental health, it is unclear if the usage was for the purpose of treating mental health concerns. Another limitation of this study is the use of self-report measures. Since mental illness is highly stigmatized in East Asia, the self-report data might be subject to bias and underreport. Still another limitation of this study is the lack of data for Taiwan and Japan. The Japanese participants were not asked the question on social support sources, and the Taiwanese participants were not asked the mental health question. Finally, the scope of this study was limited to understanding the prevalence and country-level 
difference in mental health support sources. As a consequence, the study did not examine the mechanisms through which such support sources can influence mental health.

Notwithstanding, these limitations the findings of this study have important implications for both theory and practice. In terms of theory, the findings warranted further study on a more holistic approach in conceptualizing the overall mental health treatments. The fact that the prevalence rate of kin, non-kin, and alternative medicine are high among this particular sample is important to know because it influences how practitioners should approach East Asian patients and devise therapeutic interventions. Practically, with East Asian patients, practitioners should integrate family members, social support, and alternative medicines as part of the intervention process. Moreover, providing psychoeducation on mental illness and its treatment to family members might improve treatment outcome. On treatment implementation, practitioners should consider the cultural aspect and ensure patient confidentiality to save family's face which might improve treatment compliance and increase the professional helpseeking rate. On the macro level, the government should promote mental illness education to reduce stigma. 


\section{References}

$\mathrm{Au}, \mathrm{A}$. (2017). Low mental health treatment participation and Confucianist familial norms among East Asian immigrants: A critical review.

International Journal of Mental Health, 46(1), 1-17. doi:10.1080/00207411.2016.1264036

Busiol, D. (2016). Help-seeking behaviour and attitudes towards counselling: A qualitative study among Hong Kong Chinese university students. British Journal of Guidance \& Counselling, 44(4), 382-401.

Cabinet Office. (2008). Annual report on government measures for persons with disabilities 2008. Retrieved from http://www8.cao.go.jp/shougai/english/annualreport/2008/pdf/p111.pdf

Chen, P. W. (1999). Towards professionalism: The development of counseling in Taiwan. Asian Journal of Counseling, 6(2), 21-46.

Cheung, M., Leung, P., \& Cheung, A. (2011). Depressive symptoms and help-seeking behaviors among Korean Americans. International Journal of Social Welfare, 20(4), 421-429.

Chin, W. Y., Chan, K. T. Y., Lam, C. L. K., Lam, T. P., \& Wan, E. Y. F. (2015). Help-seeking intentions and subsequent 12-month mental health service use in Chinese primary care patients with depressive symptoms. BMJ Open, 5(1), 1-10. doi:10.1136/bmjopen-2014-006730

Cho, M. J., Kim, J. K., Jeon, H. J., Suh, T., Chung, I. W., Hong, J. P., ... Lee, C. K. (2007). Lifetime and 12-month prevalence of DSM-IV psychiatric disorders among Korean adults. The Journal of Nervous and Mental Disease, 195(3), 203-210. doi:10.1097/01.nmd.0000243826.40732.45

Cook, A. L., Lei, A., \& Chiang, D. (2010). Counseling in China: Implications for counselor education preparation and distance learning instruction. Journal for International Counseling Education, 2, 60-73. Retrieved from https://pdfs.semanticscholar.org/a9f8/ff1dff10cfef389a5ff9fc73aef5d0d 37ec7.pdf

Eguchi, S. (1991). Between folk concepts of illness and psychiatric diagnosis: Kitsune-tsuki (fox possession) in a mountain village of Western Japan. Culture, Medicine and Psychiatry, 15(4), 421-451. doi:10.1007/bf00051327 
Fabrega, H. (1991). Psychiatric stigma in non-Western societies. Comprehensive Psychiatry, 32, 534-551. https://doi.org/10.1016/0010-440X(91)90033-9

Fiedler, K (2010). China's "Christianity Fever" revisited: Towards a community-oriented reading of Christian conversions in China. Journal of Current Chinese Affairs, 39(4), 71-109.

Foundation of the Japanese Certification Board for Clinical Psychologists. (n.d.). Rinshyou shinrishi shikakunintei no joist (The implementation of the certification in clinical psychology). Tokyo, Japan: Author.

Fu, T. S., Lee, C., Gunnell, D., Lee, W., \& Cheng, A. T. (2013). Changing trends in the prevalence of common mental disorders in Taiwan: A 20-year repeated cross-sectional survey. The Lancet, 381(9862), 235241. doi:10.1016/S0140-6736(12)61264-1

Hakverdioğlu, G., \& Türk, G. (2006). Acupressure. Journal of Hacettepe University School of Nursing, 13(2), 43-47.

Hanzawa, S., Bae, J., Tanaka, H., Tanaka, G., Bae, Y. J., Goto, M., ... Nakane, Y. (2009). Family stigma and care burden of schizophrenia patients: Comparison between Japan and Korea. Asia-Pacific Psychiatry, 1(3), 120-129. doi:10.1111/j.1758-5872.2009.00039.x

Hohenshil, T. H., Amundson, N. E., \& Niles, S. G. (Eds.). (2015). Counseling around the world: An international handbook. Alexandria, VA: John Wiley \& Sons.

Hong, Young-Gi. 1999. Nominalism in Korean Protestantism. Transformation, 16(4), 135-141.

Hossain, R. (2006). Atlas: Child and adolescent mental health resources, global concerns: Implications for the future: World Health Organisation (2005). International Journal of Migration, Health and Social Care, 2(1), 67. https://doi.org/10.1108/17479894200600009

Hou, Z, \& Zhang, N. (2007). Counseling psychology in China. Applied Psychology, 56(1), 33-50. doi:10.1111/j.1464-0597.2007.00274.x

Iwai, N., Li, L., Kim, S., \& Chang, Y. (2010). East Asian Social Survey (EASS), Cross-National Survey Data Sets: Health and Society in East Asia, 2010. Ann Arbor, MI: EASSDA [distributor], Interuniversity Consortium for Political and Social Research [distributor], 2014-05-01. https://doi.org/10.3886/ICPSR34608.v2 
Iwakabe, S. (2005). Mental health and counseling in Japan: A path toward societal transformation. Journal of Mental Health Counseling, 27, 129-141. https://doi.org/10.17744/mehc.27.2.ttm606ectpw5vfed

Iwakabe, S. (2008). Psychotherapy integration in Japan. Journal of Psychotherapy Integration, 18, 103-125. doi:10.1037/10530479.18.1.103

Jamieson, S. (2004). Likert scales: How to (ab) use them. Medical Education, 38, 1217-1218. doi:10.1111/j.1365-2929.2004.02012.x

Jenni, C. B. (1999). Psychologists in China: National transformation and humanistic psychology. Journal of Humanistic Psychology, 39 (2), 26-47. doi:10.1177/0022167899392003

Jung, J. H., \& Olson, D. V. A. (2014). Religion, stress, and suicide acceptability in South Korea. Social Forces, 92(3), 1039-1059. doi.org/10.1093/sf/sot129

Kessler, R. C., Chiu, W. T., Demler, O., \& Walters, E. E. (2005). Prevalence, severity, and comorbidity of 12-month DSM-IV disorders in the National Comorbidity Survey Replication. Archives of General Psychiatry, 62(6), 617-627. doi:10.1001/archpsyc.62.6.617

Kim, A. E. 2003. Religious influences on personal and societal well-being. Social Indicators Research, 62(1-3):149-170.

Kim, K. H., Kim, Y. R., Noh, S. H., Kang, K. W., Kim, J. K., Yang, G. Y., \& Lee, B. R. (2013). Use of acupuncture for pain management in an academic Korean medicine hospital: A retrospective review of electronic medical records. Acupuncture in Medicine, 31(2), 228-234. doi: 10.1136/acupmed-2012-010257

King, Y.C., \& Bond, M. H. (1985) The Confucian paradigm of man: A sociological view. In W. S. Tseng \& Y. H. Wu (Eds), Chinese Culture and Mental Health, (pp. 29-45). London, England: Academic Press.

Kleinman, A. (1980). Patients and healers in the context of culture. Berkeley, CA: University of California Press.

Lam, C. S., Angell, B., Tsang, H. W. H., Shi, K., Corrigan, P. W., Jin, S., ... Larson, J. E. (2010). Chinese lay theory and mental illness stigma: Implications for research and practices. Journal of Rehabilitation, 76, 35-40.

Lee, S. M., Oh, I., \& Suh, S. (2007). Comparison study of Korean and American school counseling for developing a Korean school 
counseling model. Korean Journal of Counseling and Psychotherapy, 19, 539-567.

Lee, S. M., Suh, S., Yang, E., \& Jang, Y. J. (2012). History, current status, and future prospects of counseling in South Korea. Journal of Counseling \& Development, 90(4), 494-499. doi:10.1002/j.15566676.2012.00061.x

Lee, T. S. (2009). Born again: Evangelicalism in Korea. Honolulu, HI: University of Hawaii Press.

Lim, S., Lim, B., Michael, R., Cai, R., \& Schock, C. K. (2010). The trajectory of counseling in China: Past present, and future trends. Journal of Counseling \& Development, 88, 4-8. doi:10.1002/j.15566678.2010.tb00141.x

Lin, J. C. H. (2000). A comparative study on title preference, work settings, and direct services time of mental health professionals. Bulletin of Educational Psychology, 34(1), 83-102. doi:10.1002/j.15566676.2013.00101.x

Ma, S. (2011). China struggles to rebuild mental health programs. Canadian Medical Association Journal, 183(2), E89-E90.

Mellor, D., Carne, L., Shen, Y., McCabe, M., \& Wang, L. (2012). Stigma toward mental illness: A cross-cultural comparison of Taiwanese, Chinese immigrants to Australia and Anglo-Australians. Journal of Cross-Cultural Psychology, 44(3), 352-364. doi: $10.1177 / 0022022112451052$

Ministry of Education. (2011). 國民小學與國民中學班級編制及教 職員員額編制準則 (Guidelines for classroom sizes and employee numbers in public elementary and middle schools). Retrieved from http://edu.law.moe.gov.tw/LawContentDetails.aspx?id= FL008932\&KeyWorldHL =\&StyleType $=1$

Na, S., Ryder, A., \& Kirmayer, L. (2016). Toward a culturally responsive model of mental health literacy: Facilitating help-seeking among East Asian immigrants to North America. American Journal of Community Psychology, 58(1-2), 211-225.

National Police Agency. (2009). Suicide statistics 2008. Tokyo, Japan: Author.

Nooney, J., \& Woodrum, E. (2002). Religious coping and church-based social support as predictors of mental health outcomes: Testing a conceptual model. Journal for the Scientific Study of Religion, 41(2), 359-368. doi:10.1111/1468-5906.00122 
Ots, T. (1990). The angry liver, the anxious heart, and the melancholy spleen. Culture, Medicine and Psychiatry, 14(1), 21-58.

Park, S., Cho, M. J., Bae, J. N., Chang, S. M., Jeon, H. J., Hahm, B. J., ... Hong, J. P. (2012). Comparison of treated and untreated major depressive disorder in a nationwide sample of Korean adults. Community Mental Health Journal, 48(3), 363-371.

Parker, G., Gladstone, G., \& Chee, K. T. (2001). Depression in the planet's largest ethnic group: The Chinese. American Journal of Psychiatry, $158(6), 857-864$.

Picco, L., Abdin, E., Chong, S., Pang, S., Vaingankar, J., Sagayadevan, V., ... Subramaniam, M. (2016). Beliefs about help seeking for mental disorders: Findings from a mental health literacy study in Singapore. Psychiatric Services, 67(11), 1246-1253.

Qianfan, Z., \& Yingping, Z. (2011). Religious freedom and its legal restrictions in China. Brigham Young University Law Review, 2011(3), 783-818.

Saint Arnault, D. M., Gang, M., \& Woo, S. (2018). Factors influencing on mental health help-seeking behavior among Korean women: A path analysis. Archives of Psychiatric Nursing, 32(1), 120-126.

Salyers, M.P., Bosworth, H. B., Swanson, J. W., Lamb-Pagone J., \& Osher, F. C. (2000). Reliability and validity of the SF-12 Health Survey among people with severe mental illness. Medical Care, 38(11), 11411150. doi:10.1097/00005650-200011000-00008

Schomerus, G., Auer, C., Rhode, D., Luppa, M., Freyberger, H. J., Schmidt, S. (2012). Personal stigma, problem appraisal and perceived need for professional help in currently untreated depressed persons. Journal of Affective Disorders, 139(1), 94-97.

Schwadel, P., \& Falci, C. D. (2012). Interactive effects of church attendance and religious tradition on depressive symptoms and positive affect. Society and Mental Health, 2(1), 21-34.

doi:10.1177/2156869312442885

Shim, J. (2016). The relationship between the use of complementary and alternative medicine and the use of biomedical services: Evidence from East Asian medical systems Asia-Pacific Journal of Public Health, 28(1), 51-60. doi: 10.1177/1010539515613411

Tata, S. P., \& Leong, F. T. L. (1994). Individualism-collectivism, social network orientation, and acculturation as predictors of attitudes toward seeking professional psychological help among Chinese 
Americans. Journal of Counseling Psychology, 41, 280-287.

Thirthalli, J., Zhou, L., Kumar, K., Gao, J., Vaid, H., Liu, H., ... Nichter, M. (2016). Traditional, complementary, and alternative medicine approaches to mental health care and psychological wellbeing in India and China. The Lancet Psychiatry, 3(7), 660-672. doi:10.1016/s2215-0366(16)30025-6

Tsai, J.-F., \& Cho, W. C. (2011). The secular trend of suicide rate and the socio-economic, media, and climatic factors in Taiwan, 1976-2009: A population-based study. Journal of Affective Disorders, 129, 270-274. doi:10.1016/j.jad.2010.08.008

Unützer, J., Klap, R., Sturm, R., Young, A.S., Marmon, T., Shatkin, J., \& Wells, K. B. (2000). Mental disorders and the use of alternative medicine: Results from a national survey. American Journal of Psychiatry, 157(11), 1851-1857.

Vigo, Thornicroft, \& Atun. (2016). Estimating the true global burden of mental illness. The Lancet Psychiatry, 3(2), 171-178. doi:10.1016/s2215-0366(15)00505-2

Ware, J. E., Jr., Keller, S. D., \& Kosinski, M. (1995). SF-12: How to score the SF-12 physical and mental health summary scales (2nd ed.). Boston, MA: The Health Institute, New England Medical Center.

Ware, J. E., Jr., Kosinski, M., \& Keller, S. D. (1996). A 12-item short-form health survey: Construction of scales and preliminary tests of reliability and validity. Medical Care, 34, 220-233. http://dx.doi.org/10.1097/00005650-199603000-00003

Woo, J., Park, E., Lee, M., Ahn, M., Kwon, S., \& Koo, K. (2014). Changes in attitudes toward and patterns in traditional Korean medicine among the general population in South Korea: A comparison between 2008 and 2011. BMC Complementary and Alternative Medicine, 14(1), 436-442.

Xu, J., \& Yang, Y. (2009). Traditional Chinese medicine in the Chinese health care system. Health Policy, 90(2-3), 133-139. doi: 10.1016/j.healthpol.2008.09.003

Zhang, Y., Lao, L., Chen, H., \& Ceballos, R. (2012). Acupuncture use among American adults: What acupuncture practitioners can learn from National Health Interview Survey 2007. Evidence-Based Complementary and Alternative Medicine, 710-750. doi.org/10.1155/2012/710750 
Zhao, J., \& Shi, X. (2011). Investigation and analysis of the present situation of acupuncturist in China. Chinese Medical Herald, 8(18), $152-153$. 
Table 1

Frequency and Percentage of Support Sources from East Asian Social Survey

\begin{tabular}{lllllll}
\hline & \multicolumn{2}{l}{ Kin Support } & \multicolumn{2}{l}{ Non-Kin Support } & \multicolumn{2}{l}{ Professional Support } \\
\hline Rating & Frequency & $\%$ & Frequency & $\%$ & Frequency & $\%$ \\
\hline Not at all & 173 & 8.2 & 300 & 14.2 & 697 & 70.3 \\
Seldom & 340 & 16.2 & 397 & 18.8 & 157 & 15.8 \\
Sometimes & 713 & 33.9 & 853 & 40.3 & 97 & 9.8 \\
Often & 627 & 29.8 & 463 & 21.9 & 26 & 2.6 \\
Very & 252 & 12.0 & 102 & 4.8 & 14 & 1.4 \\
Often & & & & & & \\
Total & 2105 & 100 & 2115 & 100 & 991 & 100 \\
\hline
\end{tabular}


Table 2

Cross-Tabulations and Chi-square Test Results for Alternative Medicine by Country

\begin{tabular}{|c|c|c|c|c|}
\hline $\begin{array}{l}\text { Alternative } \\
\text { Medicine Type }\end{array}$ & China & Japan & $\begin{array}{l}\text { South } \\
\text { Korea }\end{array}$ & $\begin{array}{l}\text { Chi-Square Tests } \\
\text { of Independence }\end{array}$ \\
\hline Acupuncture & & & & $X^{2}(2,3)=$ \\
\hline No $n(\%)$ & $1224(84.2 \%)$ & $1041(94.0 \%)$ & $562(65.9 \%)$ & 270.33 \\
\hline Yes $n(\%)$ & $229(15.8 \%)$ & $67(6.0 \%)$ & $291(34.1 \%)$ & $p=.00$ \\
\hline Herbal & & & & $X^{2}(2,3)=$ \\
\hline medicine & & & & 266.33 \\
\hline No $n(\%)$ & $901(61.9 \%)$ & $992(89.9 \%)$ & $659(77.6 \%)$ & $p=.00$ \\
\hline Yes $n(\%)$ & $554(38.1 \%)$ & $111(10.1 \%)$ & $190(22.4 \%)$ & \\
\hline Acupressure & & & & $X^{2}(2,3)=84.49$ \\
\hline No $n(\%)$ & $1308(90.4 \%)$ & $874(77.4 \%)$ & $702(82.5 \%)$ & $p=.00$ \\
\hline Yes $n(\%)$ & $139(9.6 \%)$ & $255(22.6 \%)$ & $149(17.5 \%)$ & \\
\hline
\end{tabular}

